\title{
Immunohistochemical expression of Fascin-1 in colorectal cancer in relation to clinical and pathological parameters
}

\author{
Barbara M. Piskor ${ }^{1}$, Anna Pryczynicz ${ }^{2}$, Emilia Lubowicka', Katarzyna Miniewska ${ }^{3}$, \\ Justyna Zinczuk², Konrad Zareba ${ }^{4}$, Katarzyna Guzinska-Ustymowicz ${ }^{2}$ \\ ${ }^{1}$ Department of Esthetic Medicine, Medical University of Bialystok, Bialystok, Poland \\ ${ }^{2}$ Department of General Pathomorphology, Medical University of Bialystok, Bialystok, Poland \\ ${ }^{3}$ Clinical Research Center, Medical University of Bialystok, Bialystok, Poland \\ ${ }^{4}$ 2nd Department of General and Gastroenterological Surgery, Medical University of Bialystok, Bialystok, \\ Poland
}

\begin{abstract}
Introduction. Fascins are a group of proteins taking part in the maintenance of a proper structure of the cellular cytoskeleton. Fascin-1 is an actin-bundling protein present in neurons, fibroblasts, endothelial, smooth muscle, dendritic and mesenchymal cells whereas lack of its expression is characteristic of epithelial cells. Fascin-1 overexpression can be observed in neoplastic cells. Therefore, the aim of this study was to assess the expression of Fascin-1 protein in patients with colorectal cancer (CRC) and to analyze associations between Fascin-1 expression and clinical-pathological parameters.

Material and methods. The study material included postoperative samples (tumor and unchanged colon tissue) obtained from 51 CRC patients. Fascin-1 expression was assessed in the paraffin sections by immunohistochemistry. Results. A statistically significant correlation was found between the histological type of cancer and the expression of Fascin-1 ( $p=0.012)$. Increased expression of Fascin-1 in CRC was more frequent in adenocarcinoma type without the mucosal component with a better prognosis and decreased expression of this protein correlated with infiltration of cancer cells to blood and lymphatic vessels $(p=0.038)$.

Conclusions. Our findings indicate a potential role of Fascin-1 in the pathogenesis of colon cancer; however, further studies will show whether this protein plays a role in the infiltration of colorectal cancer cells. (Folia Histochemica et Cytobiologica 2018, Vol. 56, No. 2, 106-112)
\end{abstract}

Key words: colorectal cancer; Fascin-1; metastasis; IHC

\section{Introduction}

Cancer is a leading cause of death worldwide both in high and low developed countries. The incidence of colorectal cancer (CRC) rate varies among different regions - higher rates are observed in the developed and industrial countries like Japan, Western

\footnotetext{
Correspondence address: A. Pryczynicz, Department of General Pathomorphology, Medical University of Bialystok, Waszyngtona 13, 15-269 Bialystok, Poland tel.: +48 8574859 96; fax: +48 857485996 e-mail: pryczynicz.anna@gmail.com
}

Europe, and North America, and lower CRC rates in the less-developed countries of Eastern Europe, Latin America and Asia. In the developing countries dietary habits characterized by higher intake of fat, fast foods, meat and total calories, along with lack of physical activity, high prevalence of obesity and genetic factors cause remarkable increase in the burden of CRC. Moreover, increasing life expectancy and population growth result in longer exposure to carcinogenic factors [1].

Most frequently CRC develops on the base of adenoma found in the colon mucosa. Adenomas are benign lesions which become dysplastic lesions over time, resulting in carcinoma. Adenomas or carcino- 
mas are easy to remove in their early stage during the colonoscopy. Biological material obtained in this procedure is the valuable material for diagnostic and screening examination, which is a vital prognostic factor in case of CRC detection. Neoplastic process lasts a long time before the symptoms of the disease, therefore prophylaxis is so important as well as the search for new markers detecting cancer lesions and new therapeutic targets [2].

Neoplastic processes involve changes in cellular cytoskeleton which lead to acquiring by the cancer cells ability to migrate. The eukaryotic cellular cytoskeleton consists of microfilaments (actin filaments), microtubules and intermediate filaments. Actin is available in two forms: monomeric (G-actin) and filamentous (F-actin). G-actinin the presence of magnesium ions and ATP transforms into the F-actin. F-actin play a crucial role in many cellular functions like motility and the maintenance of cell shape, polarity and regulation of transcription. It is also involved in the formation of structures associated with cell migration, such as filopodia [3-5]. Fascins are the group of globular proteins responsible for maintenance of a proper structure of the cellular cytoskeleton and are the main actin filament-bundling proteins in filopodia. The genome of mammals codes three isoforms of fascin: Fascin-1, which occurs physiologically in neurons, fibroblasts, endothelial cells, smooth muscles cells, dendrite and mesenchymal cells; Fascin-2 found in the retina cells and Fascin-3 occurring in the testis [6-8]. In normal epithelial cells, Fascin-1 is not expressed, whereas its overexpression is observed in the neoplastic cells, including CRC cells [7, 9-11]. Fascin-1 has two F-actin binding sites. The interactions between Fascin-1 and $\mathrm{F}$-actin depend on the activity of protein kinase $\mathrm{C}$ alpha (PKC $\alpha$ ) and GTPases located in the extracellular matrix [7]. Fascin-1 binds actin filaments, organizing it in the fibers crosslinked collaterally, and also stimulates secretion of growth factors and cytokines by the cells [7]. Fascin- 1 was shown to bind $\beta$-catenin, which is responsible for adhesion of cells and belongs to a signal pathway of Wnt. Wnt is a group of proteins playing an essential role in embryogenesis and carcinogenesis [12]. The studies on cell cultures showed that Fascin-1 forms filopodia, structures responsible for cell migration [11, 13]. Fascin-1 plays a key role in these structures and is the only actin-binding protein present in the whole length of filopodia. Cells with high Fascin-1 expression are higher migratory ability and thus are more invasive [6-8, 10,11]. Jawhari et al. [11], studying in vitro epithelial colon cell, demonstrated the lack of expression of Fascin-1 in normal cells and the presence of Fascin-1 in the cells of inflammatory infiltration and cancer cells. The results of their stud- ies suggest that overexpression of Fascin-1, associated with formation of filopodia, may increase migratory capability and enhance proliferation of neoplastic cells [11].

Therefore, the aim of the present study was to evaluate the expression of Fascin- 1 in colorectal cancer and to analyze associations between Fascin-1 expression and clinical and pathological parameters.

\section{Material and methods}

Patients. The study material constituted postoperative samples obtained from 51 patients ( 34 men and 17 women) diagnosed with colorectal cancer, treated in the Second Department of General and Gastroenterological Surgery, Medical University of Bialystok (Bialystok, Poland) between 2005 and 2013. In 29 patients, carcinoma lesions were located in the rectum, whereas in 22 patients, they were found in other parts of the large intestine. Diagnosis of colorectal cancer was established based on the results of histopathological examination. The adenocarcinoma type without a mucosal component was observed in 41 patients, while the mucinous type in 10 patients. The grade of histological differentiation was moderately differentiated in case of 48 patients and poorly differentiated only in 3 patients. According to Classification of Malignant Tumors (TNM) [14], in one case, the tumor infiltrated the submucosal layer (pT1), in 3 cases, the layer of smooth muscles (pT2), in 44 cases the tumor infiltrated the subserous layer (pT3), and only in 3 cases it infiltrated surrounding organs (pT4). In 17 patients, metastases were found in surrounding lymphatic nodes, while in 10 patients, distant metastases were detected in the liver and lungs (Table 1). Additionally, the presence of tumor budding was assessed according to Guzińska-Ustymowicz [15], while the inflammatory infiltration within the tumor and embolisms of neoplastic cells in blood and lymphatic vessels were evaluated according to Jass et al. [16]. The study was performed after receiving the approval of the Bioethical Committee (R-I-002/11/2017) and appropriate written informed consent regarding the use of tissue was obtained from each patient in the study.

Immunohistochemistry. The expression of Fascin-1 was determined in the cancerous tissue and healthy mucosal tissue of the large intestine by the immunohistochemical method. The examinations were carried out on the paraffin blocks with the embedded tissues. Healthy mucosa of the large intestine constituting a control group was obtained from the fragment of the intestine without inflammation or cancer cells, resected during surgery. The tissue material was fixed in formalin and embedded in paraffin, then the tissue fragments sections were cut into $4 \mu \mathrm{m}$ thick sections and placed on silanized glasses. To prepare for staining, the sections were heated up at $60^{\circ} \mathrm{C}$ for 1 hour. The material 
was deparaffinized consecutively in xylenes and hydrated in alcohols. For antigen retrieval slides were incubated in citrate buffer $(\mathrm{pH}=6)$ at $97^{\circ} \mathrm{C}$ for $20 \mathrm{~min}$ and at room temperature (RT) for the next $20 \mathrm{~min}$. The activity of endogenous peroxidase was blocked by incubating the sections in $3 \% \mathrm{H}_{2} \mathrm{O}_{2}$ for $5 \mathrm{~min}$. Nonspecific antibody binding was blocked by the use of Protein Block (Novocastra, Warsaw, Poland) for $5 \mathrm{~min}$. Next, sections were incubated with the mouseanti-Fascin-1 antibody (clone \#833223, 1:50; R\&D Systems, Minneapolis, MN, USA) for $60 \mathrm{~min}$. at RT. The reaction was visualized with a post-primary reagent (rabbit anti mouse IgG, NovoLink Polymer, Novocastra) and DAB (3,3'-diaminobenzidine) chromogen (Novocastra). Cellular nuclei were stained with hematoxylin; next, slides were dehydrated in alcohols, rinsed in xylenes and closed in Cytoseal XYL medium (Thermo Scientific, Warsaw, Poland). Positive and negative control was performed according to the manufacturer's recommendations. Stained preparations were observed under light microscope. The expression of Fascin-1 protein was estimated quantitatively based on the percentage of immunoreactive cells. Fascin-1 expression was qualified as low at $<50 \%$ of positively staining cancer cells, and as high at $\geq 50 \%$ of positively staining cancer cells. At the magnification of the microscope $\times 200$, five view fields were chosen, in which the percentage of positively staining cancer cells was counted and averaged.

Statistical analysis. Statistical analysis was based on STATISTICA 12.0 program (StatSoft, Tulsa, OK, USA). Spearman's correlation test was used to determine relations between Fascin-1 protein expression and clinical and pathological parameters. The value $\mathrm{p}<0.05$ was regarded as statistically significant. Missing data were removed in pairs.

\section{Results}

The clinical-pathological characteristics of the patients are presented in Table 1.

No expression of Fascin-1 protein was observed in normal colon mucosa (Fig. 1A). In colorectal cancer, Fascin-1 expression was revealed in the cytoplasm of cancer cells (Fig. 1B, 1C). Low expression of Fascin-1 protein was found in $25.5 \%$ of cases, while high in $74.5 \%$. Statistical analysis showed lack of statistically significant correlations between Fascin-1 expression and age, sex, tumor's localization, grade of histopathological differentiation, metastases to surrounding lymph nodes and distant metastases, depth of cancer cell infiltration in the intestinal wall, inflammatory infiltration and the presence of tumor budding. However, a statistically significant correlation was proven between the histological type of a tumor and Fascin-1 expression $(\mathrm{p}=0.012, \mathrm{R}=-0.3508)$. A high expression of Fascin-1 more frequently occurred in adeno-
Table 1. Characteristics of the study group

\begin{tabular}{|c|c|}
\hline Parameter & No. of cases $(\%)$ \\
\hline \multicolumn{2}{|l|}{ Age } \\
\hline$<60$ & $9(17.6)$ \\
\hline$\geq 60$ & $42(82.4)$ \\
\hline \multicolumn{2}{|l|}{ Sex } \\
\hline Male & $34(66.7)$ \\
\hline Female & $17(33.3)$ \\
\hline \multicolumn{2}{|l|}{ Localization } \\
\hline Colon & $29(56.9)$ \\
\hline Rectum & $22(43.1)$ \\
\hline \multicolumn{2}{|c|}{ Adenocarcinoma type } \\
\hline Nonmucinosum & $41(80.4)$ \\
\hline Mucinosum & $10(19.6)$ \\
\hline \multicolumn{2}{|c|}{ Grade of malignancies } \\
\hline 2 & $48(94.1)$ \\
\hline 3 & $3(5.9)$ \\
\hline \multicolumn{2}{|l|}{ pT stage } \\
\hline 1 & $1(1.9)$ \\
\hline 2 & $3(5.9)$ \\
\hline 3 & $44(86.3)$ \\
\hline 4 & $3(5.9)$ \\
\hline \multicolumn{2}{|c|}{ Lymph node metastasis } \\
\hline Absent & $34(66.7)$ \\
\hline Present & $17(33.3)$ \\
\hline \multicolumn{2}{|l|}{ Distant metastasis } \\
\hline Absent & $41(80.4)$ \\
\hline Present & $10(19.6)$ \\
\hline \multicolumn{2}{|l|}{ Vascular invasion } \\
\hline Absent & $28(56.0)$ \\
\hline Present & $22(44.0)$ \\
\hline \multicolumn{2}{|c|}{ Inflammatory infiltration } \\
\hline 0 & $14(28.0)$ \\
\hline 1 & $16(32.0)$ \\
\hline 2 & $11(22.0)$ \\
\hline 3 & $9(18.0)$ \\
\hline \multicolumn{2}{|l|}{ Tumor budding } \\
\hline Absent & $19(38.0)$ \\
\hline Present & $31(62.0)$ \\
\hline
\end{tabular}

Missing data were removed in pairs

carcinoma without the mucosal component (80.49\%) than in mucocellular cancer $(50 \%)$. A weak negative correlation was shown between cancer cell invasion to blood and lymphatic vessels and lack of Fascin-1 


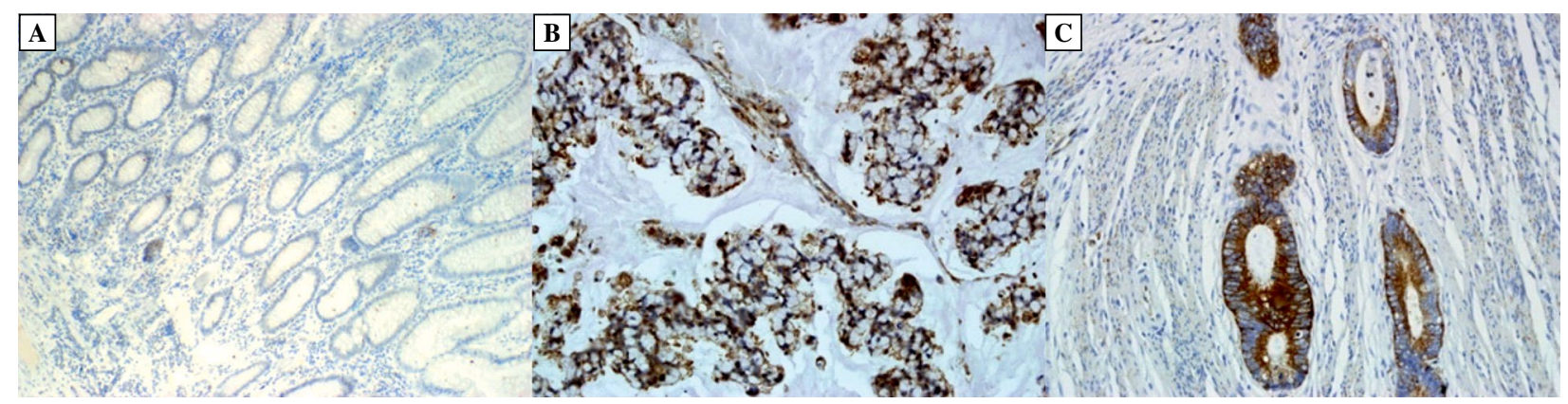

Figure 1. Expression of Fascin-1 protein in colorectal cancer. A. Lack of Fascin-1 immunoreactivity in normal colon mucosa. B. Positive expression of Fascin-1 in the signet ring cells of colorectal cancer. C. Fascin-1 immunoreactivity in crypt-like glands of colorectal cancer. Immunohistochemical staining of Fascin-1 was performed as described in Methods. Magnification $200 \times$

expression ( $p=0.038, R=-0.2944)$. In CRC patients without infiltration of cancerous cells to the blood and lymphatic vessels, a higher expression of Fascin-1 was observed, while a lower expression of this protein was revealed in the vascular invasion (Table 2).

\section{Discussion}

Colorectal cancer, as one of the most common carcinomas, constitutes an important challenge for researchers investigating its pathomechanisms and clinicians. More specific and sensitive markers of neoplastic processes are continuously being searched, which would enable early detection of cancer lesions. Furthermore, scientists seek new therapeutic targets that would improve life quality of CRC patients. In patients with diagnosed CRC, researchers frequently look for specific proteins in serum and tissue bioptates. Numerous data report about Fascin-1, a protein maintaining the structure of cytoskeleton, as a new potential marker whose expression is found in many neoplasms derived from epithelial cells [17, 18]. Cancer cells are able to metastasise after obtaining the phenotype that facilitates their movement and migration by the reconstruction of cell core actin filamentous network and formation of cell processes called filopodia [19, 20]. This process is accompanied by Fascin-1 transcription [17]. Vingjevic et al. [21] analyzed function of fascin during filopodia formation in cell cultures by using RNAi technique. Fascin silencing in cell cultures caused $4-5$ fold decrease in the number of filopodia as well as abnormal morphology and organization of actin bundles. Moreover, it was found that other actin filaments cross-linkers like -actinin or fibrin could not fully substitute for fascin function in vivo. Additionally, these authors produced point mutants mimicking the active or inactive dephosphorylated serine39 (S39E) states of fascin and reported that S29E mutants also inhibited filopodia formation. The results of this study strongly suggest that fascin is a specific actin cross-linker and plays a crucial role in maintenance stiffness and proper elongation and bundling of filopodial actin filaments [21].

It was reported that cell lines derived from adenocarcinoma of the colon with Fascin-1 expression were characterized by higher invasiveness and metastasizing capacity after transplantation [20], whereas the same cell types with a decreased expression of Fascin-1 had a reduced capability of migration and adhesion [22]. In the animal study, Schoumacher et al. [23] proved that conditional expression of Fascin-1 in the intestinal epithelium of mice with the Apc-mutated background resulted in a lower survival rate and a higher risk of the tumor growth, accelerated cancer progression and led to transformation into invasive adenocarcinoma in the intestines [23].

The expression of Fascin-1 was also studied in other cancers of the gastrointestinal tract. Li et al. [24] showed Facin-1 presence of in $95 \%$ of pancreatic ductal adenocarcinoma cells. In this study, the high histoscore significantly correlated with a lower patients' survival rate, invasion to blood and lymphatic vessels and advanced staging of cancer. Kim et al. [25] studied Fascin-1 expression in stomach cancer and found that it was associated with patients' older age, male sex, depth of tumor invasion, presence of metastases to lymph nodes, advanced staging of tumor and its invasion to the blood and lymphatic vessels [25].

The expression of Fascin-1 was also demonstrated in other types of cancers. Similarly, in the studies of breast cancer, Yoder et al. [26] proved that increased Fascin-1 expression negatively correlated with patients' survival. They found no correlation with metastases to lymph nodes, to distant organs or size of the tumor. In this group of patients, Fascin-1 ex- 
Table 2. Correlations between immunohistochemical expression of Fascin-1 in tumor tissue and clinical-pathological parameters in colorectal cancer patients

\begin{tabular}{|c|c|c|c|c|}
\hline \multirow[t]{2}{*}{ Parameter } & \multicolumn{4}{|c|}{ Fascin-1 expression } \\
\hline & Low $<50 \%(\%)$ & High $\geq 50 \%(\%)$ & $R$ coefficient & $P$ value \\
\hline \multicolumn{5}{|l|}{ Age } \\
\hline$<60$ & $2(22.22)$ & $7(77.78)$ & \multirow{2}{*}{-0.0567} & \multirow{2}{*}{0.693} \\
\hline$\geq 60$ & $11(26.19)$ & $31(72.81)$ & & \\
\hline \multicolumn{5}{|l|}{ Sex } \\
\hline Male & $8(23.53)$ & $26(76.47)$ & \multirow{2}{*}{-0.0865} & \multirow{2}{*}{0.542} \\
\hline Female & $5(29.41)$ & $12(70.59)$ & & \\
\hline \multicolumn{5}{|l|}{ Localization } \\
\hline Colon & $7(24.14)$ & $22(75.86)$ & \multirow{2}{*}{-0.0671} & \multirow{2}{*}{0.640} \\
\hline Rectum & $6(27.27)$ & $16(72.73)$ & & \\
\hline \multicolumn{5}{|c|}{ Adenocarcinoma type } \\
\hline Nonmucinosum & $8(19.51)$ & $33(80.49)$ & \multirow{2}{*}{-0.3508} & \multirow{2}{*}{0.012} \\
\hline Mucinosum & $5(50.00)$ & $5(50.00)$ & & \\
\hline \multicolumn{5}{|c|}{ Grade of malignancies } \\
\hline 2 & $13(27.08)$ & $35(72.92)$ & \multirow{2}{*}{0.1602} & \multirow{2}{*}{0.272} \\
\hline 3 & $0(0.00)$ & $3(100)$ & & \\
\hline \multicolumn{5}{|l|}{ pT stage } \\
\hline 1 & $0(0.00)$ & $1(100)$ & \multirow{4}{*}{-0.0431} & \multirow{4}{*}{0.764} \\
\hline 2 & $1(33.33)$ & $2(66.67)$ & & \\
\hline 3 & $11(25.00)$ & $33(75.00)$ & & \\
\hline 4 & $1(33.33)$ & $2(66.67)$ & & \\
\hline \multicolumn{5}{|c|}{ Lymph node metastasis } \\
\hline Absent & $7(20.59)$ & $27(79.41)$ & \multirow{2}{*}{-0.1770} & \multirow{2}{*}{0.214} \\
\hline Present & $6(35.29)$ & $11(64.71)$ & & \\
\hline \multicolumn{5}{|l|}{ Distant metastasis } \\
\hline Absent & $11(26.83)$ & $30(73.17)$ & \multirow{2}{*}{-0.0438} & \multirow{2}{*}{0.765} \\
\hline Present & $2(20.00)$ & $8(80.00)$ & & \\
\hline \multicolumn{5}{|l|}{ Vascular invasion } \\
\hline Absent & $4(14.29)$ & $24(85.71)$ & 07044 & 0030 \\
\hline Present & $9(40.91)$ & $13(59.09)$ & & 0.050 \\
\hline Inflammatory infil & & & & \\
\hline 0 & $5(35.71)$ & $9(64.29)$ & & \\
\hline 1 & $4(25.00)$ & $12(75.00)$ & 01735 & 0228 \\
\hline 2 & $3(27.27)$ & $8(72.73)$ & $0.1 / 35$ & $0.2 \angle 0$ \\
\hline 3 & $1(11.11)$ & $8(88.89)$ & & \\
\hline Tumor budding & & & & \\
\hline Absent & $3(15.79)$ & $16(84.21)$ & 0 1044 & 0176 \\
\hline Present & $10(32.26)$ & $21(67.74)$ & $-0.17+0$ & 0.170 \\
\hline
\end{tabular}

The expression of Fascin-1 was evaluated on the immunohistochemically stained sections as described in Methods. Spearman's correlation coefficient test was used. Missing data were removed in pairs.

pression correlated with a negative status of estrogen and progesterone receptors. Additionally, multifactor analysis indicates that Fascin-1 may be an important prognostic factor in this type of cancer [26]. In ovar- 
ian cancer a high expression of Fascin-1 was related to more advanced staging of cancer, a low stage of histopathological differentiation and a low survival rate in patients with mucinous cystadenocarcinoma [27]. Lee et al. [28] showed that presence of Fascin-1 in squamous cancer of the oral cavity had a prognostic value and its expression correlated with metastases to lymph nodes, a total survival rate and a tumor's growth. All these studies confirm that Fascin-1 influences the neoplastic process in epithelial cells.

In multifactorial analyses in advanced colorectal cancer it was found that Fascin-1 might be an independent prognostic factor [29]. Also other authors documented correlations between Fascin-1 expression in CRC and clinical and pathological parameters [30-33]. In our study, no expression of Fascin-1 was observed in the normal cells of colon mucosa, which is in accordance with the similar results obtained in the studies of other authors [29-31,33]. The relationship between the histological type of the tumor and increased expression of Fascin-1 was noted. More frequently the presence of this protein was observed in adenocarcinoma cells without the mucosal component. Similarly, Puppa et al. [29] and Hashimoto et al. [30] reported that more frequent expression of Fascin-1 was found in the same type of cancer. Moreover, the presence of Fascin-1 in CRC cells correlated with advanced dysplasia and a high stage of histopathological differentiation and a tumor's size [32]. Fascin-1 immunoreactivity scores were significantly increased in CRC with metastases to lymph nodes and advanced staging of cancer [33].

Puppa et al. [29] suggested that patients at the same advancement stage of CRC could be divided into categories of the risk of remission and progression of the disease according to the Fascin-1 expression. They showed a correlation between Fascin-1 expression and sex, tumor grade and metastases to lymph nodes and distant metastases [29]. In our study, no correlation was found between Fascin-1 expression and age, sex, tumor location and a grade of histopathological differentiation, metastases to surrounding lymph nodes and distant metastases, depth of cancer cell infiltration in the intestinal wall, inflammatory infiltration and the presence of tumor budding. Our results may be a consequence of study limitations (study group size, contingency).

Our study provides an observation between decreased expression of Fascin-1 and invasiveness of cancer cells to lymphatic and blood vessels. Kim et al. [25] showed a relation between the expression of Fascin-1 in gastric cancer and lymphovascular invasion. Moreover, Yamamoto et al. [34] showed a significant correlation between expression of Fascin-1 in gastrointestinal stromal tumors and blood vessel invasion. Since our results are contrary to the other reports about the role of Fascin-1 overexpression in the invasiveness and migration of cancer cells, it is essential to perform further thorough analyses of this problem.

Our studies show that increased expression of Fascin-1 was more common in the unfavorable clinical type of adenocarcinoma without mucosal component which suggests a potential role of this protein in the histogenesis of colon cancer.

\section{References}

1. Torre LA, Siegel RL, Ward EM, et al. Global Cancer Incidence and Mortality Rates and Trends--An Update. Cancer Epidemiol Biomarkers Prev. 2016; 25(1): 16-27, doi: 10.1158/1055-9965.EPI-15-0578, indexed in Pubmed: 26667886.

2. Marley AR, Nan H. Epidemiology of colorectal cancer. Int J Mol Epidemiol Genet. 2016; 7(3): 105-114, indexed in Pubmed: 27766137.

3. Fletcher DA, Mullins RD. Cell mechanics and the cytoskeleton. Nature. 2010; 463(7280): 485-492, doi: 10.1038/nature08908, indexed in Pubmed: 20110992.

4. Dominguez R, Holmes KC. Actin structure and function. Annu Rev Biophys. 2011; 40: 169-186, doi: 10.1146/annurev-biophys-042910-155359, indexed in Pubmed: 21314430.

5. Stricker J, Falzone T, Gardel ML. Mechanics of the F-actin cytoskeleton. J Biomech. 2010; 43(1): 9-14, doi: 10.1016/j. jbiomech.2009.09.003, indexed in Pubmed: 19913792.

6. Hashimoto Y, Skacel M, Adams JC. Roles of fascin in human carcinoma motility and signaling: prospects for a novel biomarker? Int J Biochem Cell Biol. 2005; 37(9): 1787-1804, doi: 10.1016/j.biocel.2005.05.004, indexed in Pubmed: 16002322.

7. Adams JC. Roles of fascin in cell adhesion and motility. Curr Opin Cell Biol. 2004; 16(5): 590-596, doi: 10.1016/j. ceb.2004.07.009, indexed in Pubmed: 15363811.

8. Jayo A, Parsons M. Fascin: a key regulator of cytoskeletal dynamics. Int J Biochem Cell Biol. 2010; 42(10): 1614-1617, doi: 10.1016/j.biocel.2010.06.019, indexed in Pubmed: 20601080.

9. Kanda Y, Kawaguchi T, Kuramitsu Y, et al. Fascin regulates chronic inflammation-related human colon carcinogenesis by inhibiting cell anoikis. Proteomics. 2014; 14(9): 1031-1041, doi: 10.1002/pmic.201300414, indexed in Pubmed: 24574163.

10. Kulasingam V, Diamandis EP. Fascin-1 is a novel biomarker of aggressiveness in some carcinomas. BMC Med. 2013; 11:53, doi: 10.1186/1741-7015-11-53, indexed in Pubmed: 23443037.

11. Jawhari AU, Buda A, Jenkins M, et al. Fascin, an actin-bundling protein, modulates colonic epithelial cell invasiveness and differentiation in vitro. Am J Pathol. 2003; 162(1): 69-80, doi: 10.1016/S0002-9440(10)63799-6, indexed in Pubmed: 12507891.

12. Cheng M, Xue H, Cao W, et al. Receptor for Activated C Kinase 1 (RACK1) Promotes Dishevelled Protein Degradation via Autophagy and Antagonizes Wnt Signaling. J Biol Chem. 2016; 291(24): 12871-12879, doi: 10.1074/jbc.M115.708818, indexed in Pubmed: 27129200.

13. Li A, Dawson JC, Forero-Vargas M, et al. The actin-bundling protein fascin stabilizes actin in invadopodia and potentiates protrusive invasion. Curr Biol. 2010; 20(4): 339-345, doi: 10.1016/j.cub.2009.12.035, indexed in Pubmed: 20137952. 
14. Sobin LH, Gospodarowicz MK, Wittekind Ch. TNM classification of malignant tumours. Wiley-Blackwell. Oxford 2009.

15. Guzińska-Ustymowicz K. Tumor budding in colorectal cancer: recent progress in colorectal cancer research. Tetsuichiro Muto E, Mochizuki H, Masaki T.New York: Nova Biomedical Books. Chapter: Aggressive and non-aggressive tumor budding in colorectal cancers. ; 2006: 105-116.

16. Jass JR, Love SB, Northover JM. A new prognostic classification of rectal cancer. Lancet. 1987; 1(8545): 1303-1306, indexed in Pubmed: 2884421.

17. Ma Y, Machesky LM. Fascin1 in carcinomas: Its regulation and prognostic value. Int J Cancer. 2015; 137(11): 2534-2544, doi: 10.1002/ijc.29260, indexed in Pubmed: 25302416.

18. Tan VY, Lewis SJ, Adams JC, et al. Association of fascin-1 with mortality, disease progression and metastasis in carcinomas: a systematic review and meta-analysis. BMC Med. 2013; 11: 52, doi: 10.1186/1741-7015-11-52, indexed in $\mathrm{Pu}-$ bmed: 23442983.

19. Yamashiro S, Yamakita Y, Ono S, et al. Fascin, an actin-bundling protein, induces membrane protrusions and increases cell motility of epithelial cells. Mol Biol Cell. 1998; 9(5): 993-1006, indexed in Pubmed: 9571235.

20. Vignjevic D, Schoumacher M, Gavert N, et al. Fascin, a novel target of beta-catenin-TCF signaling, is expressed at the invasive front of human colon cancer. Cancer Res. 2007; 67(14): 6844-6853, doi: 10.1158/0008-5472.CAN-07-0929, indexed in Pubmed: 17638895 .

21. Vignjevic D, Kojima Si, Aratyn Y, et al. Role of fascin in filopodial protrusion. J Cell Biol. 2006; 174(6): 863-875, doi: 10.1083/jcb.200603013, indexed in Pubmed: 16966425.

22. Hashimoto Y, Parsons M, Adams JC. Dual actin-bundling and protein kinase C-binding activities of fascin regulate carcinoma cell migration downstream of Rac and contribute to metastasis. Mol Biol Cell. 2007; 18(11): 4591-4602, doi: 10.1091/mbc.E07-02-0157, indexed in Pubmed: 17855511.

23. Schoumacher M, El-Marjou F, Laé M, et al. Conditional expression of fascin increases tumor progression in a mouse model of intestinal cancer. Eur J Cell Biol. 2014; 93(10-12): 388-395, doi: 10.1016/j.ejcb.2014.08.002, indexed in Pubmed: 25269996.

24. Li A, Morton JP, Ma Y, et al. Fascin is regulated by slug, promotes progression of pancreatic cancer in mice, and is associated with patient outcomes. Gastroenterology. 2014;
146(5): 1386-13896.e1, doi: 10.1053/j.gastro.2014.01.046, indexed in Pubmed: 24462734.

25. Kim SuJ, Kim DC, Kim MC, et al. Fascin expression is related to poor survival in gastric cancer. Pathol Int. 2012; 62(12): 777-784, doi: 10.1111/pin.12012, indexed in Pubmed: 23252866.

26. Yoder BJ, Tso E, Skacel M, et al. The expression of fascin, an actin-bundling motility protein, correlates with hormone receptor-negative breast cancer and a more aggressive clinical course. Clin Cancer Res. 2005; 11(1): 186-192, indexed in Pubmed: 15671545.

27. Lin $\mathrm{Ck}, \mathrm{Su} \mathrm{HY}$, Tsai WC, et al. Association of cortactin, fascin-1 and epidermal growth factor receptor (EGFR) expression in ovarian carcinomas: correlation with clinicopathological parameters. Dis Markers. 2008; 25(1): 17-26, indexed in Pubmed: 18776588.

28. Lee TK, Poon RTP, Man K, et al. Fascin over-expression is associated with aggressiveness of oral squamous cell carcinoma. Cancer Lett. 2007; 254(2): 308-315, doi: 10.1016/j. canlet.2007.03.017, indexed in Pubmed: 17499430.

29. Puppa G, Maisonneuve P, Sonzogni A, et al. Independent prognostic value of fascin immunoreactivity in stage III-IV colonic adenocarcinoma. Br J Cancer. 2007; 96(7): 1118-1126, doi: 10.1038/sj.bjc.6603690, indexed in Pubmed: 17375048.

30. Hashimoto Y, Skacel M, Lavery IC, et al. Prognostic significance of fascin expression in advanced colorectal cancer: an immunohistochemical study of colorectal adenomas and adenocarcinomas. BMC Cancer. 2006; 6: 241, doi: 10.1186/14712407-6-241, indexed in Pubmed: 17029629.

31. Oh SY, Kim YB, Suh KW, et al. Prognostic impact of fascin-1 expression is more significant in advanced colorectal cancer. J Surg Res. 2012; 172(1): 102-108, doi: 10.1016/j. jss.2010.07.015, indexed in Pubmed: 20851411.

32. Tsai WC, Chao YC, Sheu LF, et al. Overexpression of fascin- 1 in advanced colorectal adenocarcinoma: tissue microarray analysis of immunostaining scores with clinicopathological parameters. Dis Markers. 2007; 23(3): 153-160, indexed in Pubmed: 17473384.

33. Ozerhan IH, Ersoz N, Onguru O, et al. Fascin expression in colorectal carcinomas. Clinics (Sao Paulo). 2010; 65(2): 157-164, doi: 10.1590/S1807-59322010000200007, indexed in Pubmed: 20186299.

34. Yamamoto H, Kohashi K, Fujita A, et al. Fascin-1 overexpression and miR-133b downregulation in the progression of gastrointestinal stromal tumor. Mod Pathol. 2013; 26(4): 563-571, doi: 10.1038/modpathol.2012.198, indexed in Pubmed: 23196799.

Submitted: 14 December, 2017

Accepted after reviews: 2 June, 2018

Available as AoP: 7 June, 2018 